\title{
UM MODELO ANIMAL DE COMUNICAÇÃO INTERPESSOAL DE ESTADOS INTEROCEPTIVOS (PRIVADOS)
}

\author{
AN ANIMAL MODEL OF THE INTERPERSONAL COMMUNICATION OF \\ INTEROCEPTIVE (PRIVATE) STATES
}

\author{
DAVID LUBinski E Travis ThOMPSON \\ UNIVERSIDADE DE MINNESOTA
}

\begin{abstract}
RESUMO
Pombos foram ensinados a interagir comunicativamente (i.e., trocar estímulos discriminativos) baseados no estado interno de um deles, que variava em função da administração de cocaína, pentobarbital e salina. Esses desempenhos foram generalizados para agentes farmacológicos não treinados ( $d$-anfetamina e clorodiazepoxina) e foram observados na ausência de estimulação aversiva, privação e reforçamento incondicionado. O procedimento de treino utilizado neste estudo parece similar àquele pelo qual humanos aprendem a relatar (tatear) seus ambientes internos e talvez se constitua em um modelo animal rudimentar da comunicação interpessoal de eventos privados.
\end{abstract}

Palavras-chave: eventos privados, tatos, comunicação interanimal, emoção, pombos.

\begin{abstract}
Pigeons were taught to interact communicatively (i.e., exchange discriminative stimuli) based on 1 pigeon's internal state, which varied as a function of cocaine, pentobarbital, and saline administration. These performances generalized to untrained pharmacological agents ( $d$-amphetamine and chlordiazepoxide) and were observed in the absence of aversive stimulation, deprivation, and unconditioned reinforcement. The training procedure used in this study appears similar to that by which humans learn to report on (tact) their internal environments and may be construed as a rudimentary animalmodel of the interpersonal communication of private events.

Keywords: private events, tacts, interanimal communication, emotion, pigeons.
\end{abstract}

Roger Schnaitter (1978) definiu eventos privados como "aqueles fenômenos de interesse psicológico que têm lugar 'dentro da pele', em um nível encoberto, observáveis além da primeira pessoa, se é que o são, por meios indiretos" (p. 1). O presente experimento investigou o papel de eventos privados experimentalmente manipulados no estabelecimento da ocasião para comportamentos comunicativos entre organismos não humanos. Os objetivos deste estudo foram determinar se animais não humanos poderiam aprender a interagir comunicativamente (i.e., trocar estímulos discriminativos um com o outro), com base em eventos em seu meio interno, relatar com base em eventos internos (privados) similares que não tivessem sido envolvidos no treino e se o desempenho nessas tarefas poderia ser observado na ausência de uma operação estabelecedora primária (i.e., sem estimulação aversiva ou privação) e de reforço incondicionado.

Já é claro o fato de que é possível ensinar chimpanzés (Fouts, 1973; Gardner \& Gardner, 1971; Rumbaugh, 1977) e gorilas (Patterson, 1978) a interagir por meio da troca de estímulos discriminativos que sejam emparelhados arbitrariamente com algum as-

\footnotetext{
${ }^{1}$ Lubinski, D., \& Thompson, T. (1987). An animal model of the interpersonal communication of interoceptive (private) states. Journal of the Experimental Analysis of Behavior, 48,1-15. Tradução de Júlio C. de Rose. Revisão de Renato Bortoloti.
} 
pecto de seu ambiente externo. Embora haja discordância sobre se tais intercâmbios constituem uma verdadeira atividade linguística (Brown, 1970; Premack \& Woodruff, 1978; Terrace, Petitto, Sanders, \& Bever, 1979), há pouca dúvida de que os elementos básicos da representação simbólica do significado (Gardner \& Gardner, 1984) e rudimentos da semântica infantil humana primitiva tenham sido ensinados para primatas não humanos (Gardner \& Gardner, 1975; Savage-Rumbaugh, 1984). Por exemplo, Savage-Rumbaugh, Rumbaugh e Boysen (1978) ensinaram chimpanzés a usar estímulos pictóricos para relatar a outros chimpanzés a presença ou a ausência de objetos reais em uma sala próxima, à qual apenas um dos animais tinha acesso visual.Além disso, elementos limitados de tal intercâmbio interanimais foram também ensinados a pombos (Epstein, Lanza, \& Skinner, 1980; Lubinski \& MacCorquodale, 1984). O presente estudo desenvolveu-se a partir desse trabalho, substituindo estímulos exteroceptivos por estímulos interoceptivos privados como fonte estimuladora para o intercâmbio entre animais.

Este estudo é baseado em descobertas recentes que revelaram que animais de laboratório podem ser condicionados a emitir tatos. Um tato é definido como "um operante verbal no qual uma resposta de uma dada forma é evocada (ou pelo menos fortalecida) por um objeto ou evento particular, ou por uma propriedade de um objeto ou evento" (Skinner, 1957, pp. 81-82). Como operantes verbais, os tatos não são mantidos por reforçadores particulares e nem covariam com o estado de privação ou estimulação aversiva do sujeito; eles são frequentemente mantidos por reforçadores condicionados generalizados (i.e., estímulos que eram inicialmente neutros mas aos quais foi conferido valor reforçador por meio do pareamento com dois ou mais reforçadores incondicionados) (cf. Catania, 1984; MacCorquodale, 1969; Segal, 1977; Skinner, 1957; Winokur, 1976). Tatos são distinguidos de mandos (uma classe mais primitiva de comportamento verbal), que são definidos como "um operante verbal no qual a resposta é reforçada por uma consequência característica e está, portanto, sob controle funcional de condições relevantes de privação ou estimulação aversiva" (Skinner, 1957, pp. 35-36).

Operantes verbais satisfazendo os requisitos formais de tatos foram condicionados tanto em chimpanzés (Savage-Rumbaugh, 1984) quanto em pombos (Lubinski \& MacCorquodale, 1984). Contudo, os sujeitos nesses estudos foram treinados a tatear estímulos exteroceptivos (figuras geométricas, desenhos, cores, letras etc.). Na presente pesquisa, animais não humanos foram condicionados a tatear estímulos interoceptivos. Os tatos deles foram reforçados com um reforçador condicionado generalizado: uma luz piscante pareada com os reforçadores comida e água (tanto sob as condições relevantes de privação para cada reforçador quanto saciados).

Skinner $(1953,1957,1974)$ e outros exploraram o papel que os eventos privados podem desempenhar na modulação do comportamento manifesto (verbal e não verbal) (cf. Moore, 1980, 1984; Schnaitter, 1978). Em um dos seus artigos mais importantes, Skinner (1945) forneceu uma interpretação da maneira pela qual os seres humanos aprendem a relatar eventos privados (que não são acessíveis à comunidade verbal para confirmação). Contudo, o estudo sistemático de laborató- 
rio sobre estímulos interoceptivos regulando comportamento tem suas raízes em trabalho anterior de Pavlov e colegas (Razran, 1961). Subsequentemente, vários investigadores demonstraram que animais podem aprender a responder diferencialmente ao estado interno produzido por uma droga psicoativa por oposição ao meio interno associado com uma injeção de veículo (usualmente solução salina) (Schuster \& Brady, 1964; Thompson \& Pickens, 1971). Nos estudos típicos de discriminação de drogas com não humanos, um animal privado de comida recebe uma injeção de uma droga de treino (e.g., morfina) e dá-se a ele a oportunidade de pressionar uma de duas alavancas, ou bicar uma de duas chaves, que conduz ao reforçamento com comida (a alavanca de droga), ou uma segunda alavanca, que não produz reforçamento (a alavanca de veículo). Em dias intervenientes, o animal recebe uma injeção de veículo, e nesses dias a outra resposta é definida como correta e pressões à alavanca de droga não são reforçadas. Esse procedimento conduz a uma rápida aprendizagem de responder somente à alavanca correlacionada com droga nos dias em que a droga de treino tenha sido administrada, e de responder na alavanca alternativa nos dias em que o veículo tenha sido administrado. Um conjunto de drogas psicoativas produz responder diferencial com base em condições de estimulação interoceptiva (Colpaert, 1978; Griffiths, Roache, Ator, Lamb, \& Lukas, 1985; Holtzman, 1985; Overton, 1977). Além disso, por meio do uso, como composto de treino, de uma droga conhecida como ocupando um neuroreceptor específico, esses métodos têm permitido uma identificação rápida e precisa dos tipos de receptores neuroquímicos ocupados por uma droga de teste, o que tem sido confirmado por ensaios de isolamento de tecido (Woods, Young, \& Herling, 1982).

No presente estudo, pombos foram treinados a bicar diferencialmente três chaves em relação a salina ou uma de duas drogas, por meio do uso de um procedimento similar ao descrito por France e Woods (1985). Os pássaros foram então ensinados a intercambiar estímulos discriminativos arbitrários correlacionados com o agente que tivesse sido administrado em um dado dia. Em uma segunda fase, foi testada a generalização para estados internos de droga relacionados (e os estímulos discriminativos exteroceptivos associados). Finalmente, os intercâmbios comunicativos foram avaliados na ausência de uma operação estabelecedora primária e de reforçamento incondicionado.

\section{Método}

\section{Sujeitos}

Os sujeitos foram cinco pombos white Carneau (Columba livia) fêmeas, experimentalmente ingênuos, divididos em dois grupos, referidos como os "mandadores" (dois pombos) e "tateadores" (os três restantes). Embora o comportamento de nossos sujeitos não vá atender a todos os aspectos definidores de mandos e tatos a não ser mais tarde no experimento, nós nos referiremos a eles como mandadores e tateadores desde o início. Todos os animais foram alojados em gaiolas individuais, localizadas em um viveiro constantemente iluminado, com temperatura mantida a $25 \pm 1^{\circ} \mathrm{C}$. Cascalho era constantemente disponível na gaiola viveiro, e comida e água eram disponíveis após cada sessão, de acordo com o esquema discutido a seguir. 


\section{Equipamento}

$\mathrm{O}$ equipamento experimental foi o mesmo já utilizado na investigação de Lubinski e MacCorquodale (1984), mas modificado de várias maneiras. Ele consistia em duas câmaras experimentais contíguas, separadas por uma divisória de acrílico transparente de $0,64 \mathrm{~cm}$ de espessura, com um painel de res- postas e apresentação de estímulos individualizado (ver Figura 1). As dimensões de cada um dos dois compartimentos eram 34,3 por 31,6 por $35,6 \mathrm{~cm}$. A parede posterior de cada câmara, de alumínio, continha vários discos translúcidos (ver Figura 1) que, quando bicados com uma força de pelo menos $0,20 \mathrm{~N}$, interrompiam os contatos da chave, o que era

Câmara dos mandadores

Câmara dos tateadores
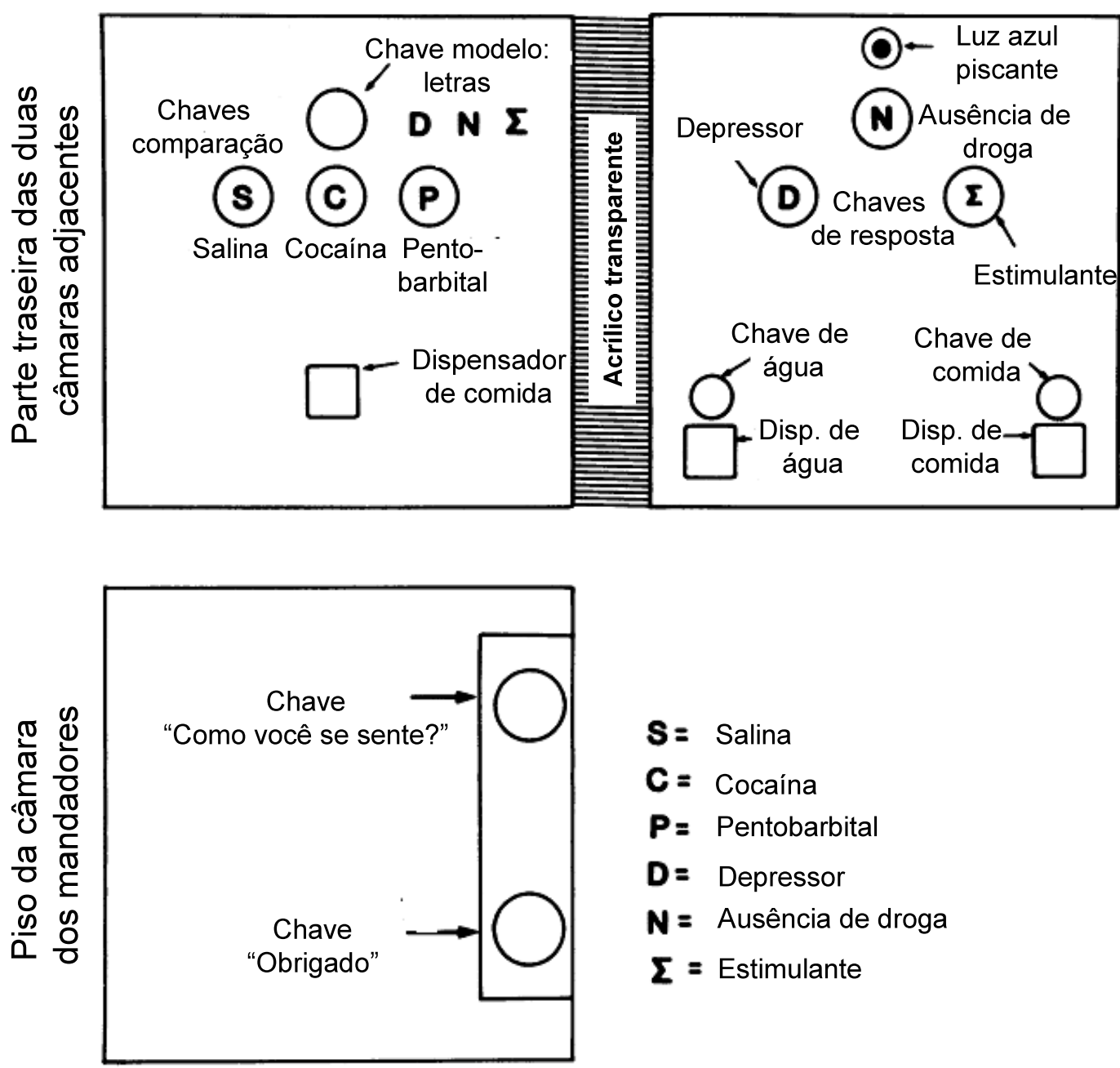

Figura 1. Os teclados adjacentes para os dois grupos de pássaros estavam separados por uma divisória de acrílico transparente. Os mandadores eram treinados na câmara da esquerda; os tateadores eram treinados na câmara da direita. $\mathrm{O}$ procedimento é apresentado na Tabela 1. 
contado como uma bicada no disco. As chaves eram transiluminadas com luzes coloridas (6 W, $110 \mathrm{vac}$ ) nos momentos indicados (ver adiante). Os estímulos eram apresentados (e todas as respostas registradas) por meio de equipamento eletromecânico localizado em uma sala próxima. Sons estranhos eram atenuados por um ventilador em operação contínua, e um mecanismo de liberação operado por solenoide foi usado para reforçamento com comida e água. Sessões experimentais eram conduzidas sete dias por semana. Os mandadores foram treinados na câmara da esquerda, e os tateadores, na da direita. $\mathrm{O}$ desempenho de um dos dois papéis (i.e., mandador ou tateador, mostrado na Tabela 1) por cada sujeito foi primeiro condicionado individualmente de acordo com o procedimento seguinte.

\section{Procedimento geral}

Treinando os tateadores. Os tateadores foram inicialmente treinados em uma cadeia complexa de dois componentes: o primeiro componente envolvia uma discriminação transmodal de emparelhamento com modelo em três chaves (composta de estímulos modelo interoceptivos emparelhados com contrapartes exteroceptivos). O segundo componente compreendia uma opção não reversível entre duas chaves (Findley, 1962), por meio da qual todas as bicadas nas chaves de comida e água eram reforçadas sob um esquema de reforçamento contínuo com seus reforçadores correspondentes.

No primeiro passo do treino dos tateadores, os pássaros, que estavam ou em privação de 28 horas de comida e 4 horas de água ou em privação de 28 horas de água e 4 horas de comida (essas duas condições alternavam-se em esquema $\mathrm{ABAB}$ ), eram treinados a bicar as chaves de comida e de água. Bicadas na chave de água tornavam disponível 0,4 ml de água por $4 \mathrm{~s}$, enquanto bicadas na chave de comida forneciam acesso a grãos misturados por 4 s. Essas respostas eram reforçadas somente quando uma luz azul estava piscando (ver Figura 1). Isso era feito para estabelecer a luz azul piscante como um reforçador generalizado condicionado (Lubinski \& MacCorquodale, 1984; Savage-Rumbaugh, 1984; Segal, 1977; Skinner, 1957). Foram requeridas aproximadamente seis semanas desde a primeira sessão de adaptação até que a luz azul piscante passasse a controlar consistentemente as bicadas dos sujeitos nas chaves de comida e água.

Os tateadores foram, então, treinados a produzir a luz azul piscante ao bicarem chaves de respostas rotuladas com letras representando "Depressor", "Estimulante" e "Ausência de droga", depois de receberem uma injeção intramuscular de, respectivamente, um depressor (pentobarbital $8 \mathrm{mg} / \mathrm{kg}$ ), ou de um estimulante (cocaína, $3 \mathrm{mg} / \mathrm{kg}$ ) ou de solução salina isotônica (administrada no mesmo volume que as outras duas soluções de droga). A solução salina também foi usada como veículo para liberação de pentobarbital e cocaína. A cocaína e o pentobarbital foram escolhidos como drogas de treino devido às suas propriedades discriminativas e reforçadoras bem documentadas para animais de laboratório (Griffiths et al., 1985; Schuster, Fischman \& Johanson, 1981) e devido à probabilidade de seu abuso por humanos (Thompson \& Unna, 1977).

Embora letras unitárias tenham sido projetadas nas chaves de resposta dos sujeitos, representando os nomes de drogas e de 
Tabela 1

O episódio verbal completo

\section{Componente 1}

Mandador: A chave "como você se sente" do mandador está iluminada e o mandador a bica, o que faz a sequência avançar para o Componente 2 .

\section{Componente 2}

Tateador: As três chaves de resposta do tateador são iluminadas simultaneamente. Ele bica a chave de resposta correspondente ao seu estado (i.e., injeção salina = "Ausência de droga", cocaína $=$ "Estimulante", pentobarbital = "Depressor"). Se o pombo bica uma chave não correspondente, a iluminação da câmara dele é apagada por $4 \mathrm{~s}$ e as condições do Componente 1 são reapresentadas. Bicar a tecla correspondente faz a sequência avançar para o Componente 3 .

\section{Componente 3}

Mandador: A tecla "obrigado" na câmara do mandador é iluminada. O mandador bica a tecla "obrigado", o que faz a sequência avançar para o Componente 4.

\section{Componente 4}

Mandador: $\quad$ O nome da droga previamente bicado pelo tateador aparece na chave de modelo do mandador.

Tateador: $\quad$ A luz azul começa a piscar na câmara do tateador.

\section{Componente 5}

Mandador: $\quad$ O mandador emparelha o nome da classe de drogas projetado na chave de modelo (i.e., ele bica a classe de drogas) com a droga apropriada (Estimulante $=$ cocaína, Depressor $=$ pentobarbital, Ausência de droga $=$ salina). Essa resposta é reforçada pelo acesso a grãos misturados. Se o mandador erra, a iluminação da câmara dele é apagada por $4 \mathrm{~s}$ e as condições do Componente 1 são reapresentadas. Ao final desse componente (i.e., depois que o mandador tenha produzido um reforçador ou ao término do timeout), a iluminação da câmara dele é apagada por $4 \mathrm{~s}$ e as condições do Componente 1 são reapresentadas, e o ciclo continua.

Tateador: $\quad$ O tateador tem acesso a grãos misturados ou água bicando a chave apropriada (depois do reforço, a luz azul para de piscar). Nos dias em que o pombo está saciado, apenas a luz piscante é apresentada; a luz azul para de piscar quando as condições do Componente 1 são reinstaladas pelo mandador. 
classes de drogas empregadas neste estudo (ver Figura 1), nós usaremos os nomes das drogas e das classes de drogas ao descrever os procedimentos, de modo a tornar esta seção mais fácil de acompanhar. Os nomes específicos apresentados nas chaves de resposta dos tateadores foram escolhidos em virtude da clareza na exposição experimental, e não como tentativa de atribuir significado simbólico.

Imediatamente após a injeção de um dos agentes anteriormente mencionados, um tateador era colocado na câmara experimental escura. Depois de um intervalo de $20 \mathrm{mi}-$ nutos, uma luz ambiente era iluminada e, simultaneamente, as três chaves de resposta eram transiluminadas. Bicadas emparelhadas ao estado interoceptivo dos pássaros (i.e., injeção salina = "Ausência de droga", pentobarbital $=$ "Depressor" e cocaína $=$ "Estimulante”) eram seguidas pela apresentação da luz azul piscante e, então, uma resposta na chave de comida ou de água era reforçada com comida ou água. Cinco bicadas eram requeridas para produzir a luz azul piscante (FR 5); se qualquer uma das outras duas chaves fosse bicada cinco vezes, a luz ambiente e a iluminação por detrás das chaves eram terminadas por $4 \mathrm{~s}$, depois do que a luz ambiente e as chaves de resposta eram reiluminadas e uma nova tentativa começava. Aproximadamente sete meses foram requeridos para que os tateadores respondessem com fidedignidade nesse procedimento de discriminação com três chaves, tanto sob condição de privação de comida quanto de água; ao longo das últimas 40 sessões desse treino de sete meses, todos os tateadores desempenharam com uma precisão geral de $90 \%$, e com pelo menos $80 \%$ de precisão em cada uma das seis condições: 2 (privação) $\times 3$ (droga) (com um mínimo de cinco observações em cada condição). Essas porcentagens são baseadas na primeira FR 5 emitida no começo de cada sessão.

Treino dos mandadores. Quatro chaves de resposta estavam diante dos mandadores em seu painel de controle: três chaves de comparação rotuladas "Pentobarbital", "Cocaína" e "Salina" e uma chave modelo sobre a qual podiam ser projetados os nomes das classes de drogas (i.e., Depressor, Estimulante e Ausência de droga). Os pesos de ambos os mandadores foram mantidos a $85 \%$ dos seus valores com comida livre durante todo o experimento. Eles foram inicialmente treinados (em uma discriminação condicional) a emparelhar as chaves de comparação rotuladas "Pentobarbital", "Salina" e "Cocaína" com os nomes das classes de droga projetados na chave modelo: "Depressor", "Ausência de droga" e "Estimulante", respectivamente. Assim, eles foram ensinados a emparelhar nomes de classes de drogas (a nomes específicos de drogas) por meio de bicadas nas chaves de resposta que continham nomes específicos de drogas.

O treino consistiu de projeção quase aleatória de nomes das três classes de droga (Depressor, Ausência de droga e Estimulante) sobre a chave modelo dos mandadores. Respostas de bicar a chave modelo quando um nome de classe de droga era projetado sobre ela e, então, de bicar a chave de resposta contendo a resposta correta de emparelhamento (i.e., Depressor $=$ Pentobarbital, Estimulante $=$ Cocaína e Ausência de droga $=$ Salina) eram reforçadas com $4 \mathrm{~s}$ de acesso a grãos misturados. Depois que os pássaros 
tornaram-se proficientes nessa tarefa em um nível de 90 a 95\%, dois requisitos adicionais foram acrescentados: os mandadores podiam produzir os nomes das classes de droga sobre a chave modelo apenas por meio de (primeiro) bicadas em uma chave iluminada localizada no nível do assoalho (Figura 1), rotulada "Como você se sente?" e (segundo) bicando uma chave no chão, rotulada "Obrigado".

O desempenho de mandar envolvia uma cadeia de três componentes: o elo terminal era uma tarefa de emparelhamento arbitrário com modelo, enquanto os dois componentes precedentes (primeiro, bicar a chave "Como você se sente?" e, segundo, bicar a chave "Obrigado") eram reforçados sob um esquema de reforçamento contínuo.

Neste ponto da sequência de treino, os sujeitos individuais de ambos os grupos haviam adquirido independentemente o repertório de componentes necessário para uma interação interanimal baseada nos estados interoceptivos dos tateadores. Os tateadores e mandadores individuais foram colocados simultaneamente em suas câmaras, e o acesso a cada componente do intercâmbio comunicativo foi tornado contingente ao comportamento do outro pássaro (ver Tabela 1). (Antes de colocar os pássaros no equipamento experimental para desempenhar essa interação, foi conduzida uma manipulação de adaptação. Pares de pássaros, compostos de um mandador e um tateador, foram colocados conjuntamente em suas respectivas câmaras, com apenas as luzes ambiente e o ventilador de atenuação de ruídos operando. Cada sessão de adaptação durou 45 minutos, e todos os pássaros experienciaram pelo menos quatro de tais sessões.)

\section{FASE 1:A INTERAÇÃO INTERANIMAL}

\section{Procedimento}

A sequência de interação começava quando o mandador bicava sua chave "Como você se sente?”. Essa resposta iluminava os nomes das classes de droga nas três chaves de resposta do tateador (i.e., "Depressor", "Ausência de droga" e "Estimulante"). O tateador, então, bicava a chave de resposta correspondente à droga que ele havia recebido. Essa resposta iluminava a chave "Obrigado" na câmara do mandador. Quando o mandador bicava a chave "Obrigado", dois eventos aconteciam concorrentemente: a luz azul do tateador começava a piscar e o nome da classe de droga previamente bicado pelo tateador aparecia na chave modelo do mandador. A partir desse ponto, o restante da sequência de respostas dos dois pássaros era independente. Com a luz azul piscando, o tateador podia receber comida ou água, bicando a chave apropriada, e o mandador podia receber comida por meio do emparelhamento correto da droga específica (entre suas chaves de resposta comparação) à classe de droga (na sua chave modelo) (ver Tabela 1).

Antes de cada interação, os mandadores recebiam 12 tentativas de aquecimento no emparelhamento de nomes de classes de droga a nomes específicos de drogas, antes que o tateador fosse colocado na câmara adjacente. Cada mandador desempenhou um número igual de interações. Embora ambos os mandadores trabalhassem com todos os tateadores, um deles trabalhou primariamente com o Tateador 1, e o outro, com o Tateador 3; o Tateador 2 trabalhou 50\% do tempo com cada um dos mandadores. Para indivíduos 
em ambos os grupos, a precisão dos pássaros não variou em função de com quem eles estavam interagindo. (Antes da interação interanimal, o Tateador 3 começou a mostrar alguns sinais de tolerância ao pentobarbital. No meio e no final do período de 40 tentativas, ele começou a bicar tanto as chaves correspondentes a salina quanto a pentobarbital, tomando alguns timeouts; foi, portanto, decidido aumentar sua dose desse agente para 12 $\mathrm{mg} / \mathrm{kg}$. Nós devemos a Sheldon Sparber a recomendação dessa modificação. Depois da implementação dessa mudança, a precisão do Tateador 3 permaneceu alta através das sessões de 40 tentativas.)

Em dias alternados, os tateadores estavam privados de comida por 28 horas e de água por 4 horas ou de água por 28 horas e de comida por 4 horas. A administração das drogas ou de apenas salina foi quase aleatória: os tateadores ganhavam livre acesso tanto à comida quanto à água por 12 horas em seguida a uma sessão de cocaína ou de pentobarbital, e nenhum desses agentes foi administrado mais de duas vezes em sucessão (descontando dias com apenas salina) sem que o outro fosse administrado uma vez. A cada dia experimental, os três tateadores recebiam injeções diferentes. Essa medida foi tomada para impedir que os mandadores discriminassem qual a condição de droga que estava operando, porque, a cada dia experimental, um dos mandadores desempenharia duas vezes, e o outro, apenas uma (exceto nos dias em que foram conduzidas sessões com apenas dois tateadores: nesses dias, cada mandador desempenhou apenas uma vez). Cada sessão continuava até que o tateador ganhasse 40 reforçadores (seja comida ou água). Finalmente, os primeiros dois dias do intercâmbio interanimal não são rela- tados na Tabela 2 (ver adiante); ambos consistiram de injeções de salina (uma sob privação de comida e a outra sob privação de água) e foram considerados como dias de aquecimento (i.e., um período para os pássaros se adaptarem a trabalhar interativamente).

\section{Resultados e discussão}

Ao longo dos 40 dias experimentais, os sujeitos desempenharam essa interação com um alto grau de precisão; todos os três tateadores desempenharam com 90\% ou mais de precisão, sob todos os três estados interoceptivos (ver Tabela $2 \mathrm{~A}$ ). Um responder randômico geraria uma precisão de aproximadamente 33\%. Somente o primeiro intercâmbio de cada sessão é relatado, porque, embora os pássaros tivessem de desempenhar 40 intercâmbios interanimal por dia para manter o desempenho, uma vez que eles tivessem recebido comida ou água, o estímulo discriminativo para o responder correto não era mais apenas o estado interno induzido por droga do tateador. A precisão do desempenho dos tateadores em tentativas subsequentes à primeira variou de 95 a 99\% ao longo de todas as condições; desvios-padrão dessas percentagens variaram de 2,1 a 6,2\% (ver Apêndice 1). A precisão do desempenho dos mandadores em cada conjunto de 12 tentativas de aquecimento não diferiu sistematicamente do desempenho nas tentativas subsequentes, nas quais o tateador estava presente.

A correspondência geral entre mandadores e tateadores (i.e., ambos os pássaros desempenhando uma discriminação correta no primeiro intercâmbio interanimal) variou de 70 a 100\% de precisão. A probabilidade de uma correspondência correta acontecer ao 
Tabela 2

Porcentagem de respostas corretas para cada pombo e a correspondência entre elas na primeira troca interanimal em três fases. A Fase 1 apresenta quatro colunas de dados obtidos a partir da primeira tentativa em 40 sessões experimentais: as frações abaixo de "Privação" representam a proporção de respostas corretas dos tateadores em cada uma das seis condições -2 (privação de comida e água) $\times$ 3 (cocaína, pentobarbital e salina). O denominador é o número total de sessões para cada condição, e o numerador é o número total de "tatos" corretos de estados internos induzidos por drogas. Total de corretas (\%), somando as duas condições de privação para as três condições de droga, é a coluna seguinte. A terceira coluna apresenta a precisão do mandador ao emparelhar estímulos discriminativos de drogas específicas a estímulos discriminativos de classes de drogas (obtida a partir da primeira tentativa nas três condições em dias em que as primeiras respostas dos tateadores foram correlatas).

Finalmente, a última coluna reflete a porcentagem de correspondência correta entre os dois pombos para cada estado induzido por droga (i.e., o produto da precisão do mandador e da precisão do tateador na primeira tentativa em cada condição). Fase 2: desempenhos combinados dos três tateadores e dos dois mandadores (obtidos a partir da primeira tentativa em 48 sessões experimentais). As colunas de frações têm o mesmo significado que as colunas de frações da Fase 1, mas se referem à administração de clordiazepoxido, $d$-anfetamina e salina. Fase 3: as colunas nessa tabela têm os mesmos significados das colunas da Fase 2. Uma coluna adicional, chamada de "Saciedade", apresenta o desempenho em 18 sessões dos tateadores em estado de saciedade (duas sessões em estado de saciedade para cada uma das três condições -clordiazepoxido, $d$-anfetamina e salina - para os três pombos)

\begin{tabular}{|c|c|c|c|c|c|}
\hline \multicolumn{6}{|c|}{ FASE 1} \\
\hline \multirow{2}{*}{ Droga } & \multicolumn{2}{|c|}{ Privação } & \multirow{2}{*}{$\begin{array}{c}\text { Total de } \\
\text { corretas } \\
(\%)\end{array}$} & \multirow{2}{*}{$\begin{array}{c}\text { Precisão do } \\
\text { mandador } \\
(\%)\end{array}$} & \multirow{2}{*}{$\begin{array}{c}\text { Precisão da } \\
\text { correspondência } \\
(\%)\end{array}$} \\
\hline & Comida & Água & & & \\
\hline \multicolumn{6}{|l|}{ Tateador 1} \\
\hline Cocaína 3 mg/kg & $5 / 5$ & $4 / 5$ & 90 & 89 & 80 \\
\hline Pentobarbital $8 \mathrm{mg} / \mathrm{kg}$ & $5 / 5$ & $5 / 5$ & 100 & 90 & 90 \\
\hline Salina & $9 / 10$ & $9 / 10$ & 90 & 94 & 85 \\
\hline \multicolumn{6}{|l|}{ Tateador 2} \\
\hline Cocaína 3 mg/kg & $5 / 5$ & $4 / 5$ & 90 & 95 & 86 \\
\hline Pentobarbital $8 \mathrm{mg} / \mathrm{kg}$ & $5 / 5$ & $5 / 5$ & 100 & 100 & 100 \\
\hline Salina & $9 / 10$ & $10 / 10$ & 90 & 95 & 90 \\
\hline \multicolumn{6}{|l|}{ Tateador 3} \\
\hline Cocaína 3 mg/kg & $5 / 5$ & $5 / 5$ & 100 & 70 & 70 \\
\hline Pentobarbital $12 \mathrm{mg} / \mathrm{kg}$ & $5 / 5$ & $5 / 5$ & 100 & 80 & 80 \\
\hline Salina & $10 / 10$ & $9 / 10$ & 95 & 95 & 90 \\
\hline
\end{tabular}


(continuação)

\begin{tabular}{|c|c|c|c|c|c|c|}
\hline \multicolumn{7}{|c|}{ FASE 2} \\
\hline \multirow[t]{2}{*}{ Droga } & \multicolumn{3}{|c|}{$\begin{array}{c}\text { Privação do } \\
\text { tateador }(\mathrm{n}=3)\end{array}$} & \multirow{2}{*}{$\begin{array}{c}\text { Total de } \\
\text { corretas } \\
(\%)\end{array}$} & \multirow{2}{*}{$\begin{array}{l}\text { Precisão do } \\
\text { mandador } \\
(\%)\end{array}$} & \multirow{2}{*}{$\begin{array}{c}\text { Precisão da } \\
\text { correspondência } \\
(\%)\end{array}$} \\
\hline & Comida & Água & & & & \\
\hline Anfetamina $2 \mathrm{mg} / \mathrm{kg}$ & $5 / 6$ & $6 / 6$ & & 92 & 91 & 84 \\
\hline Clordiazepoxido $8 \mathrm{mg} / \mathrm{kg}$ & $6 / 6$ & $6 / 6$ & & 100 & 92 & 92 \\
\hline Salina & $10 / 12$ & $11 / 12$ & & 88 & 95 & 84 \\
\hline \multicolumn{7}{|c|}{ FASE 3} \\
\hline \multirow{2}{*}{ Droga } & \multicolumn{2}{|c|}{$\begin{array}{c}\text { Privação do } \\
\text { tateador }(\mathrm{n}=3)\end{array}$} & \multirow{2}{*}{$\begin{array}{l}\text { Sacie- } \\
\text { dade }\end{array}$} & \multirow{2}{*}{$\begin{array}{l}\text { Total de } \\
\text { corretas } \\
(\%)\end{array}$} & \multirow{2}{*}{$\begin{array}{l}\text { Precisão do } \\
\text { mandador (\%) }\end{array}$} & \multirow{2}{*}{$\begin{array}{c}\text { Precisão da } \\
\text { correspondência } \\
(\%)\end{array}$} \\
\hline & Comida & Água & & & & \\
\hline Anfetamina $2 \mathrm{mg} / \mathrm{kg}$ & $2 / 3$ & $2 / 3$ & $5 / 6$ & 75 & 95 & 71 \\
\hline Clordiazepoxido $8 \mathrm{mg} / \mathrm{kg}$ & $3 / 3$ & $3 / 3$ & $6 / 6$ & 100 & 92 & 92 \\
\hline Salina & $14 / 15$ & $14 / 15$ & $6 / 6$ & 92 & 97 & 89 \\
\hline
\end{tabular}

acaso era de aproximadamente 11\% (o produto da probabilidade de ambos os desempenhos individuais acontecerem ao acaso, ou seja, $0,33 \times 0,33=0,11)$. Aproximadamente 15 minutos eram requeridos para se completarem 40 intercâmbios interanimal, através de todas as condições de droga (ver Figura 2).

Embora isso possa representar uma instância singular de intercâmbio comunicativo (via tarefas de emparelhamento arbitrário com modelo) entre dois organismos não humanos com base no estado interoceptivo de um dos participantes, tais intercâmbios são familiares entre humanos. As pessoas frequentemente perguntam umas às outras sobre como elas se sentem e mantêm a probabilidade futura de relatar estados internos por meio de expressões de interesse, preocupação ou entusiasmo sobre o sentimento autorrelatado do falante. No presente experimento, as respostas de bicar dos mandadores solicitavam informação dos tateadores com base no estado interno dos últimos; os tateadores re- forçavam a solicitação dos mandadores tateando (i.e., bicando) o nome da classe de droga (em chaves iluminadas) que correspondia ao estado corrente deles; os mandadores, então, reforçavam esses tatos emitindo uma resposta que produzia a luz azul piscante e, assim, emparelhavam o relato dos tateadores bicando uma segunda classe de estímulos arbitrários que correspondiam às drogas específicas.

Embora a precisão dos pássaros tenha permanecido alta nos 40 dias experimentais, alguns aspectos do desempenho deles modificaram-se gradualmente durante essa fase. (Os sujeitos foram observados por circuito fechado de televisão durante todas as sessões.) Inicialmente, os pássaros passavam quase todo o tempo na área imediatamente adjacente às chaves de resposta e então bicavam apropriadamente quando a iluminação aparecia. Contudo, gradualmente, as atividades gerais dos pássaros pareceram ficar sob controle de estímulos fornecidos pelo comportamento do outro, assim como dos estí- 


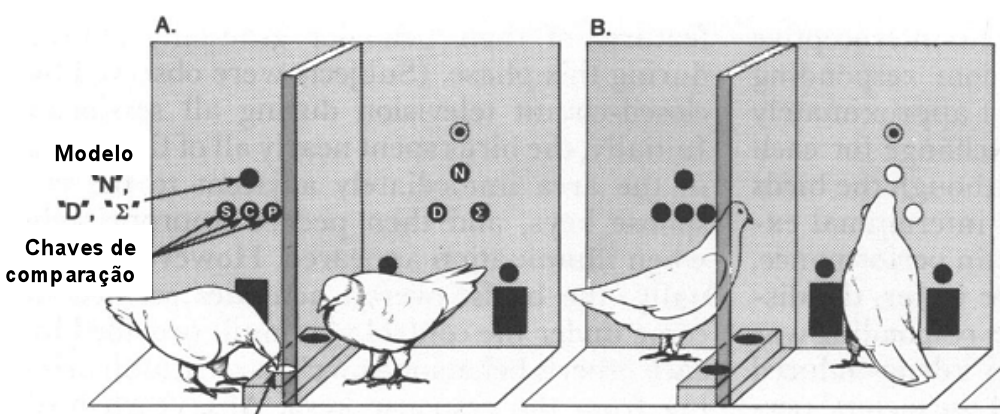

Chave "como você se sente?"

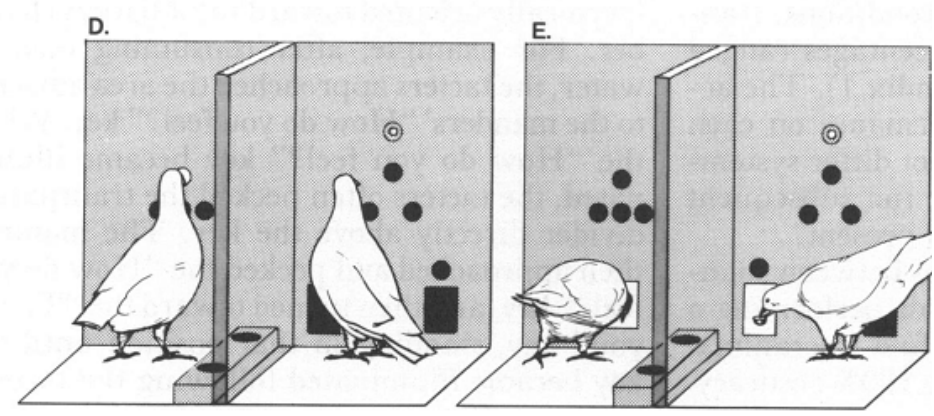

c.

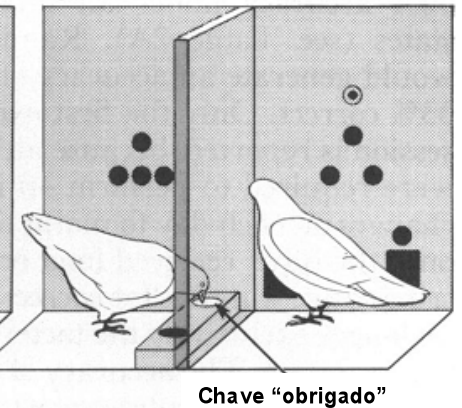

A) Mandador bica a chave "como você se sente?".

B) Tateador bica o símbolo para a classe de drogas correspondente ao seu estado.

C) Mandador bica a chave "obrigado".

D) Mandador bica o símbolo para a classe de drogas na chave modelo e o símbolo específico da droga; a luz azul piscante é apresentada ao tateador.

E) Mandador é recompensado com comida por completar corretamente a troca simbólica; tateador é reforçado com água.

Figura 2. Uma troca comunicativa entre dois pombos baseada no estado interno de um deles (mandador, à direita; tateador, à esquerda). (A) O mandador bica a chave "como você se sente?”. (B) O tateador bica a letra para a classe de drogas correspondente ao seu estado interno. (C) O mandador bica a chave "obrigado", que apresenta a luz azul piscante ao tateador; essa resposta também apresenta ao mandador a letra da classe de drogas previamente bicada pelo tateador. (D) O mandador bica a letra da classe de drogas (projetada na sua chave de modelo) e, em seguida, bica a letra que representa a droga específica que o tateador está experienciando naquele momento; o tateador atenta para a luz azul piscante. (E) O mandador é reforçado com comida por completar corretamente a troca comunicativa; o tateador é reforçado com água.

mulos arbitrários da chave de resposta. Depois de que cada pássaro completava um elo componente da sequência, ele tipicamente orientava-se em direção à câmara adjacente. Por exemplo, depois de consumir comida ou água, os tateadores se aproximavam da área adjacente à chave "Como você se sente?" dos mandadores. Quando essa chave se iluminava, os tateadores frequentemente bicavam a divisória transparente, sobre a chave. Os mandadores, então, aproximavam-se e bicavam a chave "Como você se sente?", dirigindo-se para a chave "Obrigado" e permanecendo em posição até que a chave se iluminasse em seguida à resposta dos tateadores. Se os tateadores fossem lentos para bicar a chave de classe de droga, os mandadores tendiam a bicar a partição transparente de modo similar ao descrito por Epstein et al. (1980) e Lubinski e MacCorquodale (1984). Na prática, contudo, os pássaros, em ambos os grupos, usualmente completavam elos sucessivos da interação rapidamente, de tal modo que bicadas na partição não eram típicas; elas ocorriam, contudo, se qualquer dos pássaros fizesse uma pausa. 


\section{Fase 2: Generalização para DROGAS RELACIONADAS}

\section{Procedimento}

O objetivo seguinte desta pesquisa foi determinar se o desempenho discriminativo estabelecido se generalizaria para estados similares mas algo diferentes (i.e., eventos privados induzidos por agentes farmacológicos que os sujeitos não tivessem experienciado previamente). Clordiazepoxido (Librium) e $d$-anfetamina (Dexedrina) são usados comumente em nossa cultura tanto para fins terapêuticos quanto recreacionais (Miller et al., 1983; Szara \& Ludford, 1981). Embora a $d$-anfetamina e o clordiazepoxido difiram tanto química quanto farmacologicamente da cocaína e do pentobarbital, ambos compartilham propriedades farmacológicas com esses agentes (Gilman, Goodman, \& Gilman, 1980). Além disso, é bem estabelecido que, em preparações convencionais para discriminação de duas escolhas entre droga e salina, aquelas drogas generalizam-se para cocaína (Fischman, 1984) e pentobarbital (Ator \& Griffiths, 1983), respectivamente.

O teste de generalização requereu, contudo, uma ligeira modificação de procedimento. Visto que o clordiazepoxido é absorvido e distribuído mais lentamente do que a cocaína ou o pentobarbital, o intervalo pré-sessão, entre injeção e início da sessão, foi aumentado para 30 minutos. Os primeiros dois dias com 30 minutos de intervalo pré-sessão consistiram de injeções de salina (uma sob privação de água e a segunda sob privação de comida). Os testes de generalização de droga foram conduzidos da mesma maneira imprevisível, com a $d$-anfetamina administrada a $2 \mathrm{mg} / \mathrm{kg}$ e o clordiazepoxido, a $8 \mathrm{mg} / \mathrm{kg}$, intramuscularmente no músculo do peito.

\section{Resultados e discussão}

Para 24 tentativas, seis para cada agente de teste sob privação de água e de comida, apenas um erro ocorreu (i.e., 95,8\% corretos). Essas observações foram registradas na segunda vez em que o sujeito (Tateador 1) foi exposto à $d$-anfetamina; ele havia respondido corretamente na primeira tentativa (ver Tabela $2 \mathrm{~B}$ ). A precisão do desempenho dos tateadores em tentativas subsequentes à primeira variou de 92 a 99\% ao longo de todas as condições; o desvio-padrão médio para essas percentagens foi de 4,6\% (ver Apêndice 2).

O tateador bicou a chave apropriada à droga, relatando a seu companheiro que o estado interoceptivo engendrado pela $d$-anfetamina era mais similar ao estado de cocaína do que aos estados induzidos seja por pentobarbital, seja por salina; similarmente, ele relatou que os estímulos interoceptivos produzidos por clordiazepoxido eram mais similares a um estado de pentobarbital do que aos estados de salina ou de cocaína. A precisão de correspondência para essa condição variou de 84 a 92\% de respostas corretas - o responder ao acaso seria aproximadamente $11 \%$ correto.

A discriminação interoceptiva de três chaves, dos tateadores, generalizou-se para agentes farmacológicos conhecidos por produzir condições internas similares, mas, presumivelmente, algo diferentes (Ator \& Griffiths, 1983; Fischman, 1984). O desempenho dos tateadores é similar ao relatado por France e Woods (1985). Eles treinaram pombos a bicar três chaves de respostas correspon- 
dentes ao controle de estímulo interoceptivo da administração de morfina, naltrexona ou salina. Depois que o desempenho foi firmemente estabelecido, testes de generalização foram conduzidos (usando estímulos de teste similares, mas, apesar disso, novos, ou seja, naloxona, etilcetociclazocina, buprenorfina e pentazocina). O antagonista opiáceo naloxona se generalizou para a chave de resposta do naltrexona, e os agonistas opiáceos etilcetociclazocina, buprenorfina e pentazocina se generalizaram para a chave de resposta da morfina.

Além disso, a generalização do presente estudo, por meio de depressores e de estimulantes, das discriminações entre os estados internos de cocaína, pentobarbital e salina, é consistente com estudos em laboratório animal de discriminação/generalização (duas escolhas, droga versus salina) (Ator \& Griffiths, 1983; Brady \& Griffiths, 1977; Griffiths et al., 1985; Johanson \& Schuster, 1977). Ainda mais, esses dados estão de acordo com os dados de humanos que tinham experiência com cocaína; esses sujeitos não relataram diferenças quando injetados por via intravenosa com $10 \mathrm{mg}$ de anfetamina versus $16 \mathrm{mg}$ de cocaína (Fischman \& Schuster, 1980). Em acréscimo, humanos tratados com clordiazepoxido relataram efeitos experienciais similares àqueles produzidos pelo pentobarbital (Griffiths et al., 1985).

Embora os dados presentes sejam consistentes com trabalhos prévios, a descoberta de que um animal não humano pode aprender a desempenhar uma discriminação de três estados internos, mantida por dois reforçadores, sob duas condições distintas de privação, é nova. Outro aspecto singular desse desem- penho é que ele era contido dentro de um intercâmbio comunicativo interanimal.

\section{Fase 3: Saciação e descontinuaÇão do REFORÇAMENTO PRIMÁRIO}

O tipo de comportamento comunicativo exibido pelos tateadores pode ser qualificado como tatos, isto é, respostas verbais estabelecidas com múltiplos reforçadores e que não especificam um reforçador particular. Em humanos, diz-se frequentemente que relações de tato são mantidas somente por reforçadores condicionados generalizados (Lubinski \& MacCorquodale, 1984; Savage-Rumbaugh, 1984; Skinner, 1957). No presente experimento, o reforçador condicionado generalizado era a luz azul piscante dos tateadores. Para determinar se os tateadores continuariam a relatar seus estados internos quando saciados de comida e água e sem reforçamento relevante a qualquer privação (mas com a luz azul piscante funcionando como um reforçador condicionado generalizado contingente ao responder discriminativo), foi conduzida a sonda experimental adicional a seguir.

\section{Procedimento}

O mesmo procedimento descrito na Fase 2 para $d$-anfetamina e clordiazepoxido foi mantido; contudo, a cada quatro ou cinco dias, os tateadores eram colocados em suas câmaras experimentais depois de terem recebido 24 horas de acesso livre tanto a comida quanto a água. As suas chaves de respostas de comida e água ficavam inoperantes durante essa condição e, devido à sua condição sacia$\mathrm{da}$, era requerido deles que desempenhassem apenas cinco intercâmbios interanimal, em vez dos 40 usuais. 


\section{Resultados}

Quando saciados de comida e de água (Tabela 2C) e sem reforçadores consumíveis, mas com a luz azul piscante contingente ao responder correto, os tateadores continuaram a responder corretamente às solicitações dos mandadores, relatando acuradamente seus estados internos de 83 a $100 \%$ das vezes. O Tateador 3 bicou uma chave não correspondente apenas uma vez, sob anfetamina e com privação de água, e o Tateador 2 cometeu os outros dois erros para essa condição. A precisão média do desempenho dos tateadores em tentativas subsequentes à primeira, para sessões sob saciação, foi: anfetamina, 92\%; clordiazepoxido, 90\%; e salina, 90\% (ver Apêndice 3). A precisão geral da correspondência interanimal para essa condição variou de 71 a 92\% (o desempenho ao acaso produziria uma medida de precisão de aproximadamente $11 \%)$.

\section{VERIFICAÇÕES AUXILIARES}

Depois de completar-se a Fase 3 deste estudo, medidas da taxa de bicadas no disco foram obtidas para todos os três tateadores sob administração de cada um dos quatro agentes farmacológicos, assim como de salina. Essas verificações foram efetuadas sem a participação dos mandadores, usando-se o programa de treino que havia sido empregado antes da interação interanimal. Três sessões, com 30 minutos de intervalo de injeção pré-sessão (clordiazepoxido, salina, d-anfetamina), foram seguidas por quatro sessões com 20 minutos de intervalo de injeção pré-sessão (salina, pentobarbital, salina, cocaína). Todos os sete dias de teste foram conduzidos sob privação de comida, com os pássaros a $85 \%$ dos seus pesos obtidos com comida livre. As quantidades de tempo despendido para que os pássaros obtivessem 40 reforçadores (da primeira resposta correta à liberação final do comedouro) foram: $3 \mathrm{mg} / \mathrm{kg}$ de cocaína (382 s), 8 a $12 \mathrm{mg} / \mathrm{kg}$ de pentobarbital (391 s), $2 \mathrm{mg} / \mathrm{kg}$ de $d$-anfetamina (431 s), $8 \mathrm{mg} /$ $\mathrm{kg}$ de clordiazepoxido (425 s), salina (424 s). Esses tempos representam as médias interanimal para cada condição. O tempo para salina foi computado a partir dos dados coletados em todos os três dias de salina (nove pontos de dados), que podem ser vistos como a taxa base dos pássaros.

\section{Discussão}

Os pombos foram condicionados a responder discriminativamente a três estados interoceptivos distintos; eles foram, também, treinados a intercambiar comunicativamente estímulos discriminativos arbitrários como função dos seus estados. Além disso, o desempenho discriminativo dos tateadores se generalizou para condições similares de estímulos interoceptivos e, finalmente, foi também observado na ausência de uma operação estabelecedora primária e quando os reforçadores incondicionados não mais eram liberados. A tendência dos pombos para interagir dessa maneira estava diretamente relacionada com a adequação de sua experiência e a força dos seus repertórios para relatar tais eventos privados.

O comportamento dos tateadores envolvia tatear eventos privados (via um desempenho de emparelhamento com modelo no qual os estímulos modelo eram interoceptivos). Os pássaros foram condicionados a discriminar três estados internos por meio de 
bicadas em chaves de respostas rotuladas com letras, e a discriminação foi reforçada com um reforçador condicionado generalizado (um estímulo emparelhado com a liberação de comida ou de água). Mais ainda, o reforçamento do comportamento discriminativo deles não covariou com as particularidades de seus estados de privação ou de estimulação aversiva; as respostas discriminativas deles eram reforçadas sob privação tanto de água quanto de comida e também quando eles estavam saciados. Os mandadores, por outro lado, foram condicionados a emitir mandos impuros (i.e., respostas verbais controladas conjuntamente pelos estímulos discriminativos fornecidos pelos tateadores e por um estado específico de privação (comida), sendo mantidas pelo reforçador relevante à privação).

Uma diferença entre a maneira na qual nossos sujeitos aprenderam a tatear eventos privados e a hipótese de Skinner (1945, 1984) acerca de como humanos adquirem essa habilidade é o esquema de reforçamento durante o condicionamento. No presente estudo, os eventos privados experienciados pelos nossos sujeitos (i.e., estados de droga que eles foram condicionados a tatear) eram controlados com virtualmente $100 \%$ de fidedignidade e validade; o responder acurado de nossos sujeitos sobre seus estados interoceptivos foi reforçado em um esquema de reforçamento contínuo (CRF). A maneira pela qual humanos tipicamente aprendem a tatear eventos privados, de acordo com Skinner, é bem menos precisa, e o reforçamento para fazer isso é intermitente.

A persistência do responder discriminativo generalizado mantido por meio do comportamento de outro organismo sugere que a tendência dos animais para relatar experiências internas similares, embora algo diferentes, pode ser ensinada. Tecnicamente, as respostas verbais emitidas pelos tateadores durante essa fase do experimento qualificam-se como tatos estendidos. "Há muitas maneiras pelas quais um estímulo novo pode assemelhar-se a um estímulo previamente presente quando uma resposta foi reforçada, e há, portanto, vários tipos do que pode ser denominado 'tatos estendidos"” (Skinner, 1957, p. 91). Na medida em que esses resultados descrevem adequadamente a maneira pela qual humanos relatam sentimentos novos, embora similares aos já experienciados, eles são consistentes com a hipótese de Skinner $(1945,1984)$ acerca de como humanos adquirem essa habilidade: Skinner sugere que as pessoas são capazes de descrever novos sentimentos porque esses estados frequentemente compartilham aspectos com sentimentos familiares, os quais elas já tenham aprendido a relatar (i.e., tatos estendidos baseados em generalização de estímulos interoceptivos).

A maioria das unidades comportamentais componentes exibidas pelos nossos sujeitos havia sido observada em um trabalho prévio, mas não havia sido sintetizada de forma a construir um modelo animal do comportamento comunicativo humano. Catania (1983) discutiu a utilidade de tais demonstrações: "A análise comportamental começa com relações comportamentais complexas e então as fraciona em componentes. Um teste da adequação de tal análise é uma síntese experimental (e.g., Catania, 1972; Catania \& Keller, 1981)....Algumas vezes, nós começamos com conceitos a respeito dos assuntos humanos como bases para a produção de novas relações comportamentais. A síntese con- 
siste na criação, dentro do laboratório, de um desempenho que seja, em alguns aspectos, análogo ao comportamento humano fora do laboratório ... uma vez que tal fenômeno tenha sido demonstrado por meio de uma síntese comportamental, suas propriedades definidoras e sua faixa de aplicabilidade podem ser refinadas por pesquisa subsequente. O sucesso de uma síntese é, então, julgado não apenas com base nos resultados empíricos, mas também na extensão na qual a compreensão refinada do fenômeno tem implicações para as situações humanas fora do laboratório, a partir das quais o análogo laboratorial emergiu" (pp. 58-59). (Para discussões relacionadas, ver Lubinski \& Thompson, 1986; e Thompson \& Lubinski, 1986.) Das quatro classes de comportamento humano enumeradas como candidatas a uma pesquisa animal de simulação por Epstein (1984), apenas o comportamento encoberto (i.e., pensamentos, sentimentos e imagens) tem resistido à análise experimental por meio do uso de simulações animais. O presente estudo demonstra que essa classe de comportamento também é passível de análise objetiva por via da simulação com sujeitos não humanos.

As descobertas presentes sugerem que estímulos interoceptivos e eventos novos no nível do neuroreceptor são não apenas discrimináveis como podem vir a ser relatados a outros organismos. Organismos não humanos podem ser ensinados a fazer tais discriminações e a relatá-las a seus vizinhos, comunicativamente, por meio de um processo de aprendizagem, e continuarão a fazê-lo mesmo na ausência de uma operação estabelecedora primária e de um reforçador incondicionado. Ao mesmo tempo, é pouco provável que os desempenhos estudados aqui constituam atividade linguística na forma em que esse termo é usualmente entendido (Brown, 1970). Não há razão para supor que qualquer estrutura sintática seja inerente às sequências de respostas dos pombos, assim como não há qualquer razão para atribuir intencionalidade complexa aos intercâmbios comunicativos dos pássaros. Apesar disso, o caráter desses desempenhos compartilha aspectos com aqueles verificados em crianças de idade muito pequena ou em jovens severamente prejudicados (e.g., crianças com autismo) que estejam justamente começando a responder diferencialmente a seus sentimentos internos e a relatá-los (Lovaas, 1981). Essas pessoas requerem uma comunidade social para começar a aprender como relatar seus estados internos, mas seus autorrelatos frequentemente parecem rígidos e restritos a um domínio muito estreito das experiências de aprendizagem disponíveis.

Savage-Rumbaugh (1984) apontou diferenças entre o comportamento dos chimpanzés envolvidos na pesquisa dela e dos pombos do estudo de Epstein et al. (1980), diferenças essas que levantam dúvidas sobre a afirmação de que os pombos nesta investigação estivessem de fato engajados em atividade comunicativa. Os pombos, argumenta Savage-Rumbaugh (1984), não haviam sido treinados em um ambiente verbal, de modo que não se podia propriamente dizer que estivessem engajados em comportamento verbal. Mais importante ainda, ela afirma que, uma vez que as contingências impostas sobre o comportamento dos pombos tenham sido efetuadas por meio de circuitos eletrônicos, e não por outro indivíduo, o resultado não poderia ser razoavelmente caracterizado como "comunicação". As mesmas preocupações 
seriam presumivelmente aplicáveis à presente investigação, uma vez que os pássaros não foram treinados por pessoas que se comunicassem como pombos ou por pombos que se comunicassem como pessoas, assim como os intercâmbios de estímulos discriminativos não eram mediados sem a participação de circuitos eletrônicos. A comunidade verbal experimental do pombo (i.e., o outro pombo treinado independentemente) era restrita a uma faixa extremamente limitada de comportamento, conformando-se ao modo de uma troca diádica de deixas. Desse modo, a questão é: "Dada uma audiência coespecífica preparada para responder discriminativamente a uma faixa muito limitada de deixas verbais, um pombo ao qual fosse dada a oportunidade de relatar seu ambiente interno chegaria a fazê-lo, sem estar faminto nem sedento, nem tampouco recebendo comida ou água por ter assim desempenhado?" A resposta parece ser "Sim".

Nós acreditamos que o papel do circuito externo na mediação do intercâmbio é menos pertinente à questão que temos à mão. Há incontáveis exemplos de intercâmbios diádicos humanos nos quais parte das contingências entre os estímulos e as respostas dos participantes é mediada por eventos externos entre o ouvinte e o falante e sobre os quais eles não têm controle, e nós continuamos a nos referir a tais intercâmbios como "verbais". Essas manipulações de contingências variam de conexões telefônicas imperfeitas entre falante e ouvinte a mensagens deixadas em quadros de aviso de redes de computadores (BBS) que podem ser respondidas por um computador, e não por uma pessoa. A questão essencial tem a ver com as relações funcionais entre falante e ouvinte e com as variáveis controladoras, e não com a maneira pela qual as variáveis são implementadas.

Se nós, de fato, descrevemos corretamente os aspectos-chave do modo pelo qual os humanos tipicamente aprendem a relatar seu meio interno, nós podemos estar em uma melhor posição para começar a entender as diferenças individuais na habilidade das pessoas para relatar adequadamente eventos internos. No presente experimento, a habilidade dos pombos para relatar seus estados internos dependeu da adequação de suas experiências (especificamente, treino discriminativo como função de eventos internos manipulados farmacologicamente). Se o mesmo for válido para as pessoas, podem-se esperar grandes diferenças individuais na habilidade de relatar sentimentos internos, dependendo da adequação das histórias pessoais de aprendizagem discriminativa a respeito de eventos interoceptivos, que são frequentemente adquiridas sob a tutela dos pais (ou, em algumas instâncias, mais tarde na vida, por meio de aconselhamento ou psicoterapia). Em acréscimo, se assumirmos que há diferenças individuais substanciais, determinadas constitucional e/ou geneticamente, na disponibilidade de tipos de receptores sobre os quais os neurotransmissores podem agir (com os eventos afetivos correlacionados), nós poderemos esperar diferenças individuais significativas na competência para relatar experiências internas.

A noção de que eventos internos estimuladores sejam componentes críticos da emoção tem sido amplamente mantida desde William James (1890), e essa ideia recebe credibilidade tanto por evidências recentes quanto por sugestões teóricas a respeito da relação do complexo receptor GABA- 
-benzodiazepínico com a ansiedade humana (Gray, 1982; Poshivalov, 1987). Evidências advindas de procedimentos cada vez mais refinados de laboratório animal de discriminação de drogas revelam que animais podem relatar eventos no nível de neuroreceptores, tanto quanto os organismos são capazes de responder diferencialmente ao modo como cones e bastonetes na retina são ativados. Isso sugere que pode ser possível entender mais objetivamente o papel dos estados afetivos internos por meio da combinação de tecnologias de modelo animal de discriminação de droga com aquelas da química de receptores. Mais ainda, a comunicação interorganismo de certas qualidades de estados afetivos pode ser avaliada pela junção desses métodos com o domínio da comunicação interanimal. Como Skinner (1953) observou há mais de 30 anos: "A linha entre o público e o privado não é fixa. O limite se desloca com cada descoberta de uma técnica para converter eventos privados em eventos públicos... O problema da privacidade pode, portanto, ser eventualmente resolvido por meio de avanços técnicos" (p. 282).

\section{REFERÊNCIAS}

Ator, N. A., \& Griffiths, R. R. (1983). Lorazepam and pentobarbital drug discrimination in baboons: Cross-drug generalization and interaction with Ro 15-1788. Journal of Pharmacology and Experimental Therapeutics, 226, 776-782.

Brady, J.V., \& Griffiths, R. R. (1977). Drug-maintained performance procedures and the assessment of drug-abuse liability. In T. Thompson \& K. R. Unna (Eds.). Predicting dependence liability of stimulant and depressant drugs (pp. 165-184). Baltimore: University Park Press.
Brown, R. (1970). The first sentences of child and chimpanzee. In R. Brown. Psycholinguistics: Selected papers (pp. 208-231). New York: Free Press.

Catania, A. C. (1972). Concurrent performances: Synthesizing rate constancies by manipulating contingencies for a single response. Journal of the Experimental Analysis of Behavior, 17, 139145.

Catania, A. C. (1983). Behavior analysis and behavior synthesis in the extrapolation from animal to human behavior. In G. C. L. Davey (Ed.). Animal models of human behavior (pp. 51-69). Chichester, England:Wiley.

Catania, A. C. (1984). Learning (2nd ed.). Englewood Cliffs, NJ: Prentice-Hall.

Catania, A. C., \& Keller, K. J. (1981). Contingency, contiguity, correlation, and the concept of causation. In P. Harzem \& M. D. Zeiler (Eds.). Advances in analysis of behaviour: Predictability, correlation, and contiguity (Vol. 2, pp. 125-167). Chichester, England: Wiley.

Colpaert, F. C. (1978). Discriminative stimulus properties of narcotic analgesic drugs. Pharmacology Biochemistry and Behavior, 9, 863-887.

Epstein, R. (1984). Simulation research in the analysis of behavior. Behaviorism, 12, 41-59.

Epstein, R., Lanza, R. P., \& Skinner, B. F. (1980). Symbolic communication between two pigeons (Columba livia domestica). Science, 207, 543-545.

Findley, J. D. (1962). An experimental outline for building and exploring multi-operant behavior repertoires. Journal of the Experimental Analysis of Behavior, 5, 113-166.

Fischman, M. W. (1984). Behavioral pharmacology of cocaine in humans. In J. Grabowski (Ed.). Cocaine: Pharmacology, effects, and treatment of abuse (pp. 72-91). National Institute on Drug Abuse Research Monograph No. 50 (DHHS Publication No. ADM 84-1326). Washington, DC: U.S. Government Printing Office. 
Fischman, M.W., \& Schuster, C. R. (1980). Experimental investigations of the actions of cocaine in humans. In F. R. Jeri (Ed.). Cocaine: Proceedings of the interamerican seminar on medical and sociological aspects of coca and cocaine (pp. 62-75). Lima, Peru: Pacific Press.

Fouts, R. S. (1973). Acquisition and testing of gestural signs in four young chimpanzees. Sciences, 180, 978-980.

France, C. P., \& Woods, J. H. (1985). Opiate agonistantagonist interactions: Application of a threekey drug discrimination procedure. Journal of Pharmacology and Experimental Therapeutics, 234, 81-89.

Gardner, B. T., \& Gardner, R. A. (1971). Two-way communication with an infant chimpanzee. In A. M. Schrier \& F. Stollnitz (Eds.). Behavior of nonhuman primates (Vol. 4, pp. 117-184). New York: Academic Press.

Gardner, B. T., \& Gardner, R. A. (1975). Evidence for sentence constituents in the early utterances of child and chimpanzee. Journal of Experimental Psychology: General, 104, 244-267.

Gardner, R. A., \& Gardner, B. T. (1984). A vocabulary test for chimpanzees (Pan troglodytes). Journal of Comparative Psychology, 98, 381-404.

Gilman, A. G., Goodman, L. S., \& Gilman, A. (Eds.) (1980). Goodman and Gilman's The pharmacological basis of therapeutics (6th ed.). New York: Macmillan.

Gray, J. A. (1982). Precis of The neuropsychology of anxiety: An enquiry into the functions of the septo-hippocampal system. Behavioral and Brain Sciences, 5, 469-484.

Griffiths, R. R., Roache, J. D., Ator, N. A., Lamb, R. J., \& Lukas, S. E. (1985). Similarities in reinforcing and discriminative stimulus effects of diazepam, triazolam, and pentobarbital in animals and humans. In L. S. Seiden \& R. L. Balster (Eds.). Neurology and neurobiology: Behavioral pharmacology: The current status (Vol. 13, pp. 419432). New York: Liss.

Holtzman, S. G. (1985). Discriminative stimulus properties of opioids that interact with $\mathrm{mu}$, kappa and PCP/sigma receptors. In L. S. Seiden \& R. L. Balster (Eds.). Neurology and neurobiology: Behavioral pharmacology: The current status (Vol. 13, pp. 131-147). New York: Liss.

James, W. (1890). The principles of psychology. New York: Holt.

Johanson, C. E., \& Schuster, C. R. (1977). Procedures for predicting the preclinical assessment of abuse potential of psychotropic drugs in animals. In T. Thompson \& K. R. Unna (Eds.). Predicting dependence liability of stimulant and depressant drugs (pp. 203-229). Baltimore: University Park Press. Lovaas, O. I. (1981). Teaching developmentally disabled children. Baltimore: University Park Press.

Lubinski, D., \& MacCorquodale, K. (1984). "Symbolic communication" between two pigeons (Columba livia) without unconditioned reinforcement. Journal of Comparative Psychology, 98, 372-380.

Lubinski, D., \& Thompson, T. (1986). Functional units of human behavior and their integration: A dispositional analysis. In T. Thompson \& $\mathrm{M}$. D. Zeiler (Eds.). Analysis and integration of behavioral units (pp. 275-314). Hillsdale, NJ: Erlbaum.

MacCorquodale, K. (1969). B. F. Skinner's verbal behavior: A retrospective appreciation. Journal of the Experimental Analysis of Behavior, 12, 831-841.

Miller, J. D., Cisen, I. H., Gardner-Keaton, H., Hairell, A.V., Wirtz, P. W., Absdar, H. I., \& Fishburne, P. M. (1983). National survey on drug abuse: Main findings, 1982. National Institute on Drug Abuse (DHHS Publication No.ADM 83-1263). Washington, DC: U.S. Government Printing Office.

Moore, J. (1980). On behaviorism and private events. Psychological Record, 30, 459-475. 
Moore, J. (1984). On privacy, causes, and contingencies. Behavior Analyst, 7, 3-16.

Overton, D. A. (1977). Discriminable effects of anti-muscarinics: Dose response and substitution studies. Pharmacology Biochemistry and Behavior, 6, 659-666.

Patterson, F. G. (1978). The gestures of a gorilla: Language acquisition in another pongid. Brain and Language, 5, 72-97.

Poshivalov, V. P. (1987). Computerized ethological pharmacology: The new synthesis. In T. Thompson, P. B. Dews, \& J. Barrett (Eds.). Advances in behavioral pharmacology: Neurobehavioral pharmacology (Vol. 6, pp. 193-220). Hillsdale, NJ: Erlbaum.

Premack, D., \& Woodruff, G. (1978). Does the chimpanzee have a theory of mind? Behavioral and Brain Sciences, 1, 515-526.

Razran, G. (1961). The observable unconscious and the inferable conscious in current Soviet psychophysiology: Interoceptive conditioning, semantic conditioning, and the orienting reflex. Psychological Review, 68, 81-147.

Rumbaugh, D. M. (1977). Language learning by a chimpanzee: The Lana project. New York: Academic Press.

Savage-Rumbaugh, E. S. (1984). Verbal behavior at a procedural level in the chimpanzee. Journal of the Experimental Analysis of Behavior, 41, 223250.

Savage-Rumbaugh, E. S., Rumbaugh, D. M., \& Boysen, S. (1978). Symbolic communication between two chimpanzees (Pan troglodytes). Science, 201, 641-644.

Schnaitter, R. (1978). Private causes. Behaviorism, 6, $1-12$.

Schuster, C. R., \& Brady, J.V. (1964). The discriminative control of operant behavior by interoceptive stimulation (trans. into Russian). Pavlov Journal of Higher Nervous Activity, 14, 448-458.
Schuster, C. R., Fischman, M.W., \& Johanson, C. E. (1981). Internal stimulus control and subjective effects of drugs. In T. Thompson \& C. E. Johanson (Eds.). Behavioral pharmacology of human drug dependence (pp. 116-129). National Institute on Drug Abuse Research Monograph No. 37 (DHHS Publication No. ADM 81-1137). Washington, DC: U.S. Government Printing Office.

Segal, E. F. (1977). Toward a coherent psychology of language. In W. K. Honig \& J. E. R. Staddon (Eds.). Handbook of operant behavior (pp. 628653). Englewood Cliffs, NJ: Prentice-Hall.

Skinner, B. F. (1945). The operational analysis of psychological terms. Psychological Review, 52, 270 277, 291-294.

Skinner, B. F. (1953). Science and human behavior. New York: Macmillan.

Skinner, B. F. (1957). Verbal behavior. New York: Appleton-Century-Crofts.

Skinner, B. F. (1974). About behaviorism. New York: Knopf.

Skinner, B. F. (1984). The operational analysis of psychological terms. Behavioral and Brain Sciences, 7, 547-553.

Szara, S. I., \& Ludford, J. P. (1981). Benzodiazepines: A review of research results, 1980. National Institute on Drug Abuse Research Monograph No. 33 (DHHS Publication No. ADM 81-1052). Washington, DC: U.S. Government Printing Office.

Terrace, H. S., Petitto, L.A., Sanders, R. J., \& Bever, T. G. (1979). Can an ape create a sentence? Science, 206, 891-902.

Thompson, T., \& Lubinski, D. (1986). Units of analysis and kinetic structure of behavioral repertoires. Journal of the Experimental Analysis of Behavior, 46, 219-242.

Thompson, T., \& Pickens, R. (1971). Stimulus properties of drugs. New York: Appleton-CenturyCrofts. 
Thompson, T., \& Unna, K. R. (Eds.). (1977). Predicting dependence liability of stimulant and depressant drugs. Baltimore: University Park Press.

Winokur, S. (1976). A primer of verbal behavior: An operant view. Englewood Cliffs, NJ: Prentice-Hall.
Woods, J. H., Young, A. M., \& Herling, S. (1982). Classification of narcotics on the basis of their reinforcing, discriminative, and antagonistic effects in rhesus monkeys. Federation Proceedings, 41, 221-227. 


\section{Apêndice 1}

Precisão do desempenho discriminativo dos tateadores em tentativas subsequentes à primeira resposta de emparelhamento da Fase 1, em condições de privação. A primeira coluna apresenta a porcentagem média de acertos em todas as sessões, e a segunda coluna apresenta o desvio-padrão dessa porcentagem

\begin{tabular}{lcc}
\hline & $\begin{array}{c}\text { Média de respostas } \\
\text { corretas }(\%)\end{array}$ & DP \\
\hline Tateador 1 & 99 & 2,3 \\
Cocaína $3 \mathrm{mg} / \mathrm{kg}(\mathrm{n}=10)$ & 98 & 3,7 \\
Pentobarbital $8 \mathrm{mg} / \mathrm{kg}(\mathrm{n}=10)$ & 98 & 2,6 \\
$\quad$ Salina & & \\
\hline Tateador 2 & 95 & 5 \\
Cocaína $3 \mathrm{mg} / \mathrm{kg}(\mathrm{n}=10)$ & 97 & 4,1 \\
Pentobarbital $8 \mathrm{mg} / \mathrm{kg}(\mathrm{n}=10)$ & 97 & 5,9 \\
Salina $(\mathrm{n}=20)$ & & 2,1 \\
\hline Tateador 3 & 99 & 2,6 \\
Cocaína 3 mg/kg $(\mathrm{n}=10)$ & 99 & 6,2 \\
Pentobarbital $12 \mathrm{mg} / \mathrm{kg}(\mathrm{n}=10)$ & 98 & \\
Salina $(\mathrm{n}=20)$ &
\end{tabular}

\section{Apêndice 2}

Precisão do desempenho discriminativo dos tateadores em tentativas subsequentes à primeira resposta de emparelhamento da Fase 2, em condições de privação. A primeira coluna apresenta a porcentagem média de acertos em todas as sessões, e a segunda coluna apresenta o desvio-padrão dessa porcentagem

\begin{tabular}{lcc}
\hline & $\begin{array}{c}\text { Média de respostas } \\
\text { corretas }(\%)\end{array}$ & DP \\
\hline Tateador 1 & 97 & 3,6 \\
Anfetamina $2 \mathrm{mg} / \mathrm{kg}(\mathrm{n}=4)$ & 95 & 10 \\
Clordiazepoxido $8 \mathrm{mg} / \mathrm{kg}(\mathrm{n}=4)$ & 99 & 4 \\
Salina $(\mathrm{n}=8)$ & & \\
\hline Tateador 2 & 98 & 3,6 \\
Anfetamina $2 \mathrm{mg} / \mathrm{kg}(\mathrm{n}=4)$ & 92 & 13 \\
Clordiazepoxido $8 \mathrm{mg} / \mathrm{kg}(\mathrm{n}=4)$ & 97 & 5 \\
Salina $(\mathrm{n}=8)$ & & 1,3 \\
\hline Tateador 3 & 99 & 0 \\
Anfetamina $2 \mathrm{mg} / \mathrm{kg}(\mathrm{n}=4)$ & 100 & 1,1 \\
Clordiazepoxido $8 \mathrm{mg} / \mathrm{kg}(\mathrm{n}=4)$ & 99 & \\
Salina $(\mathrm{n}=8)$ & & \\
\hline
\end{tabular}


Apêndice 3

Precisão dos tateadores depois da primeira tentativa em condições de saciedade na Fase 3.

Cada tateador foi submetido a duas sessões para cada condição (anfetamina, clordiazepoxido e salina). As frações representam o número total de respostas corretas dividido pelo número total de respostas de emparelhamento; as porcentagens representam a precisão do desempenho dos tateadores

\begin{tabular}{ll}
\hline Tateador 1 & \\
Anfetamina $2 \mathrm{mg} / \mathrm{kg}$ & $4 / 4=100 \%, 4 / 4=100 \%$ \\
$\quad$ Clordiazepoxido $8 \mathrm{mg} / \mathrm{kg}$ & $\begin{array}{l}4 / 5=80 \%, 4 / 4=100 \% \\
4 / 4=100 \%, 4 / 7=57 \%\end{array}$ \\
Salina & \\
\hline Tateador 2 & $4 / 6=67 \%, 5 / 5=100 \%$ \\
$\quad$ Anfetamina $2 \mathrm{mg} / \mathrm{kg}$ & $4 / 5=80 \%, 4 / 4=100 \%$ \\
$\quad$ Clordiazepoxido $8 \mathrm{mg} / \mathrm{kg}$ & $4 / 4=100 \%, 4 / 5=80 \%$ \\
$\quad$ Salina & \\
\hline Tateador 3 & $4 / 4=100 \%, 4 / 5=80 \%$ \\
$\quad$ Anfetamina $2 \mathrm{mg} / \mathrm{kg}$ & $4 / 4=100 \%, 4 / 5=80 \%$ \\
Clordiazepoxido $8 \mathrm{mg} / \mathrm{kg}$ & $4 / 4=100 \%, 4 / 4=100 \%$ \\
Salina &
\end{tabular}

